\title{
Discrete Cosine Transformation based Image Data Compression Considering Image Restoration
}

\author{
Kohei Arai \\ Graduate School of Science and Engineering \\ Saga University, Saga City \\ Japan
}

\begin{abstract}
Discrete Cosine Transformation (DCT) based image data compression considering image restoration is proposed. An image data compression method based on the compression (DCT) featuring an image restoration method is proposed. DCT image compression is widely used and has four major image defects. In order to reduce the noise and distortions, the proposed method expresses a set of parameters for the assumed distortion model based on an image restoration method. The results from the experiment with Landsat TM (Thematic Mapper) data of Saga show a good image compression performance of compression factor and image quality, namely, the proposed method achieved $25 \%$ of improvement of the compression factor compared to the existing method of DCT with almost comparable image quality between both methods.
\end{abstract}

Keywords-Discrete Cosine Transformation; data compression; image restoration; Landsat TM

\section{INTRODUCTION}

Image data compression methods can be divided into two types, information loss-less and information lossy. The former uses image redundancy for getting high data compression ratio. On the other hand, the later ensures no image degradation by the data compression, data compression is not so high though. JPEG imagery data compression based on DCT is one of the information lossy data compression methods and is popular and widely used data compression method. Although the data compression ratio is satisfactory good, image quality degradation is severe in comparison to the other information lossy data compression methods. Due to the facts, there are block noise, mosquito noise, color distortion noise, etc. in the JPEG data compression method.

Image data compression method proposed here is based on the well-known JPEG compression method. By using image restoration methods, the aforementioned noises are removed as much as we can in the proposed image data compression method. This is the basic idea of the proposed method which allows comparatively high data compression ratio and relatively small image degradation by the data compression.

Research on image restoration is divided into those related to restoration methods and those related to methods for estimating restoration parameters from degraded images. Image restoration methods can be roughly classified into linear restoration filters and nonlinear restoration filters [1]. The former starts with the classic Wiener filter and parametric Wiener filter, which only restore the best approximation image on average, and evaluates the difference between the restored image and the original image not on the space of the original image but on the observed image. A general inverse filter, a least-squares filter with constraints, and a projection filter and a partial projection filter that may be significantly affected by noise in the restored image have been proposed [2] However, the former is insufficient for optimization of evaluation criteria, etc., and is under study.

On the other hand, the latter is essentially a method for finding a non-linear solution, so it can take only a method based on an iterative method, and various methods based on iterative methods have been tried. There are various iterative methods, but there are a stationary iterative method typified by the successive excess relaxation method (SOR method) and an irregular iterative method typified by the conjugate gradient method [3], [4], [5]. In general, the former requires a large number of iterations, but the accuracy is high, and the latter has excellent convergence, but the problem of accumulation of rounding errors is a problem. When applied to image restoration, it is necessary to pay attention to noise resistance.

On the other hand, the maximum entropy method has been proposed as an image restoration method because it can take into account constraints (or prior knowledge) and resistance to noise [6]. In addition, as a parameter estimation method, methods using stationary iteration methods such as Newton's method and quasi-Newton's method and non-stationary iterative methods such as the conjugate gradient method have already been proposed [7], [8]. Furthermore, an annealing method has been proposed [9].

From the viewpoint of image compression, the estimation of the degradation operator (restoration parameter) by image compression on the transmission side can be performed with high accuracy because the images before and after compression can be referred to. By encoding this restoration parameter and sending it to the receiving side together with the compressed image, the receiving side can restore the image deteriorated by the compression based on the decoded restoration parameter [10]. This is the basis of the compression method with image restoration proposed in this paper.

To show the effect of this method, image compression based on orthogonal expansion is taken as an example here. This paper reports that a high-quality reconstructed image can be obtained by devising the encoding of the reconstructed parameters. 
The following section d4escribes research background followed by theoretical background. Then the proposed method is described followed by experiment. After that conclusion is described together with some discussions.

\section{RESEARCH BACKGROUND}

Facsimile data compression by rearranging picture elements is proposed [11]. Data compression for archiving of Advanced Earth Observing Satellite: ADEOS data is well reported [12]. Method for image compression with a cosmetic restoration, on the other hand, is proposed [13] together with a method for image compression with cosmetic restoration [14].

Meanwhile, a study of data lossy compression using JPEG/DCT and fractal method is conducted and well reported [15]. Also, preliminary study on information lossy and lossless coding of data compression for archiving ADEOS data is conducted and well reported [16].

Method for video data compression based on space and time domain seam carving maintaining original quality when it is replayed is proposed [17]. Data hiding method which robust to run-length data compression based on lifting dyadic wavelet transformation is proposed [18]. On the other hand, method for image portion retrieval and display for comparatively large scale of imagery data onto relatively small size of screen which is suitable to block coding of image data compression is proposed and evaluated [19].

Prediction method of El Nino Southern Oscillation event by means of wavelet based data compression with appropriate support length of base function is proposed and validated with the actual data [20]. Meanwhile, method for data hiding based on Legall 5/2 (Cohen-Daubechies-Feauveau: CDF 5/3) wavelet with data compression and random scanning of secret imagery data is proposed and evaluated effectiveness and efficiency [21].

\section{PROPOSED METHOD}

\section{A. Transmission of Compressed Data and Restoration Parameters}

Compression methods that allow image quality degradation are roughly classified into predictive coding, orthogonal transform coding represented by Fourier transform, and approximate coding represented by vector quantization. Of these, the orthogonal transform coding is a compression method that can easily estimate the restoration parameter on the transmission side. In this method, image compression is realized by reducing a large dimension of an order after orthogonal transformation, but image quality is deteriorated because high-order information is lost. Consider that the image quality degradation is restored in an integrated manner. Fig. 1 shows the outline of the method.

In the past, only compressed data obtained by compressing original image data by orthogonal transform coding was transmitted. However, the method proposed this time creates the data necessary to configure the restoration filter on the transmitting side and creates the compressed data. Transmit with.

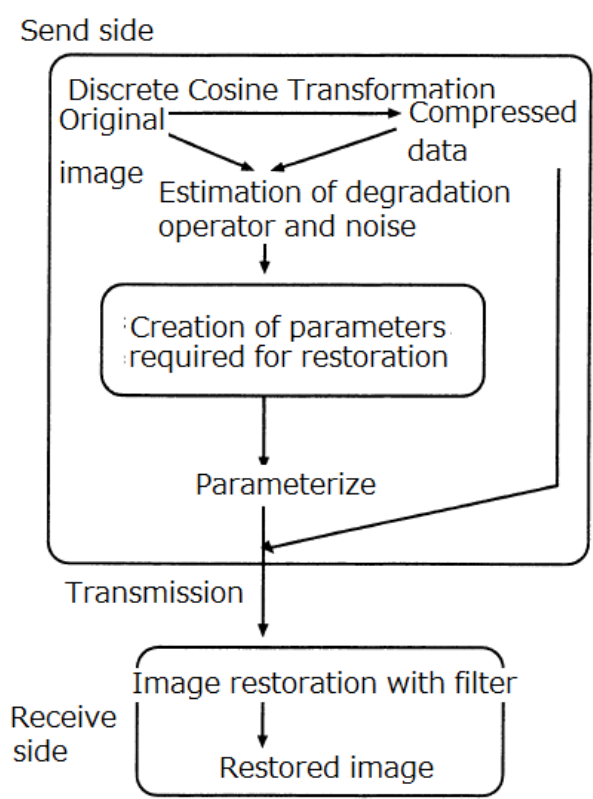

Fig. 1. Process flow of the Proposed Image Data Compression Method.

On the other hand, on the receiving side, a filter is constructed from reconstruction parameters for constructing a transmitted reconstruction filter, and a deteriorated image is restored.

\section{B. Creating a Restoration Filter}

The image quality degradation model is represented by a convolution operation as shown in equation (1).

$g(x, y)=\int_{-\infty}^{\infty} \int_{-\infty}^{\infty} h(x-\xi, y-\eta) f(\xi, \eta) d \xi d \eta+n(x, y)$

Here, $g$ represents a compressed image, $h$ represents a deterioration operator, $f$ represents an original image, and $n$ represents noise. Also, if each Fourier transform is $G, H, F, N$, then.

$G(\mu, v)=H(\mu, v) F(\mu, v)+N(\mu, v)$

Can be expressed as If the restoration filter is B $(\mu, v)$, the restoration image $\mathrm{F}(\mu, \mathrm{v})$ can be expressed by the following equation.

$\mathrm{F}(\mu, \mathrm{v})=\mathrm{B}(\mu, \mathrm{v}) \mathrm{G}(\mu, \mathrm{v})$

It suffices if $G, H, . F$, and $N$ are all known in equation (2), but the original image data and the compressed image data exist on the transmitting side, but the restoration filter and noise are unknown there. In equation (2), $G$ and $F$ are known, but $H$ and $\mathrm{N}$ are unknown. Since $\mathrm{H}$ is difficult to find, we assume $H(\mu, v)=1$ and consider a model where noise causes image quality degradation. Then, the noise $N$ is the difference between $G$ and $F$.

Then, equation (2) becomes equation (4).

$\mathrm{G}(\mu, \mathrm{v})=\mathrm{F}(\mu, \mathrm{v})+\mathrm{N}(\mu, \mathrm{v})$

It can be expressed like this. Wiener filter is considered as an example of the restoration filter.

$B(\mu, v)=\frac{H^{*}(\mu, v)}{\mid H(\mu, v)^{2}+S_{n}(\mu, v) / S_{f}(\mu, v)}$ 
$\mathrm{H}(\mu, \mathrm{v})=1, \operatorname{Sn}(\mu, \mathrm{v})=\mathrm{N}(\mu, \mathrm{v}), \operatorname{Sf}(\mu, \mathrm{v})=\mathrm{F}(\mu, \mathrm{nu})$

$B(\mu, v)=1 /\{1+\mathrm{N}(\mu, v) / F(\mu, v)$

It becomes, therefore, the following equation,

$$
\begin{aligned}
& \mathrm{F}(\mu, \mathrm{v})=\mathrm{B}(\mu, \mathrm{v}) \mathrm{G}(\mu, \mathrm{v}) \\
& =\mathrm{F}(\mu, \mathrm{v}) \mathrm{G}(\mu, \mathrm{v}) /\{\mathrm{F}(\mu, \mathrm{v})+\mathrm{N}(\mu, \mathrm{v})\} \\
& =\mathrm{F}(\mu, \mathrm{v}) \mathrm{G}(\mu, \mathrm{v}) / \mathrm{G}(\mu, \mathrm{v}) \\
& =F(\mu, v)
\end{aligned}
$$

Thus, the original image can be restored. At this time, since the deterioration operator is collected in the frequency spectrum of the S / $\mathrm{N}$ ratio, it can be restored on the receiving side only by adding this to the compressed image. The restoring filters other than the Wiener filter have many parameters of the degrading operator and are considered unsuitable for image compression.

\section{Restoration Filter Parameterization}

As an orthogonal transform, discrete cosine transform is used as an example. There have already been proposals on the spectrum estimation after conversion and the configuration of the restoration filter accompanying it [7]. Here, since the Wiener filter is used as the restoration filter, a new parameter configuration for the restoration filter is devised. The filter is expressed in equation (9).

$\mathrm{F}(\mu, \mathrm{v})=1 /\{1+\mathrm{F}(\mu, \mathrm{v}) / \mathrm{F}(\mu, \mathrm{v})\}$

Here, when looking at the portion of $N(\mu, v) / F(\mu, v)$, it can be seen that this is the reciprocal of the $\mathrm{S} / \mathrm{N}$ ratio of the original image and the noise. Therefore, it is first conceivable to parameterize the $\mathrm{S} / \mathrm{N}$ ratio for transmission. Looking at the $\mathrm{S} / \mathrm{N}$ ratio in the image, it is as shown in Fig. 2.

Fig. 3 shows the concept of $\mathrm{S} / \mathrm{N}$ ratio parameterization. In addition, when inverse quantization is performed, the maximum value (MAX) and the minimum value (MIN) of the low frequency component are required, so that these must also be transmitted. After all, what is going to be transmitted is

- SN ratio of quantized low frequency components.

- The maximum value (MAX) used for quantization of low frequency components Minimum value (MIN).

- Coefficient of equation of regression plane showing SN ratio of high frequency component.

In this figure, the difference between Fig. 4 (original image) and Fig. 5 (compressed image) is used as noise, and the $\mathrm{S} / \mathrm{N}$ ratio is shown for the red image.

It represents the $\mathrm{S} / \mathrm{N}$ ratio of the higher frequency component toward the center of the image. Also, the blacker the pixel, the lower the $\mathrm{S} / \mathrm{N}$ ratio, and the whiter the pixel, the higher the $\mathrm{S} / \mathrm{N}$ ratio.

From these observations, it can be seen that white points and black points are mixed in the low-frequency components, so that values having a considerably large absolute value are mixed, and the values vibrate violently. Conversely, it can be seen that the value of the high frequency component changes relatively smoothly. From this, it is considered difficult to parameterize the low-frequency component, so consider transmitting the low-frequency component as it is, and parameterizing and transmitting only the high-frequency component.

The above is not true for general images. However, it is not necessary to discuss that the image quality is better when the high-frequency component is approximated and sent than when the high-frequency component is deleted after the discrete cosine transform as in the existing method. What can be considered in the parameterization is a polynomial approximation. In the case of polynomial approximation, the degree of the polynomial and the coefficient of each term are transmitted. This can be determined by a method based on regression analysis.

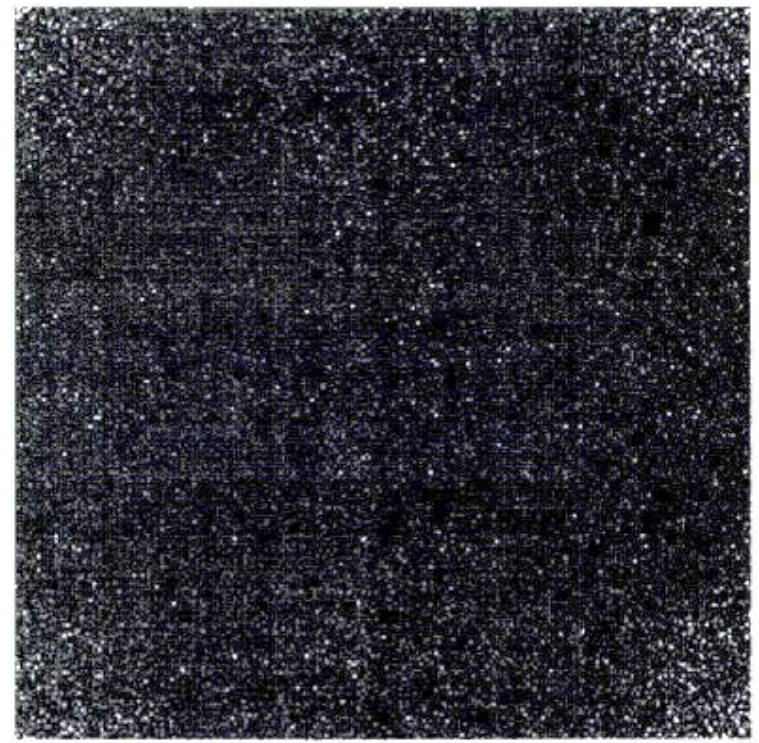

Fig. 2. S / N Ratio in the Frequency Domain.

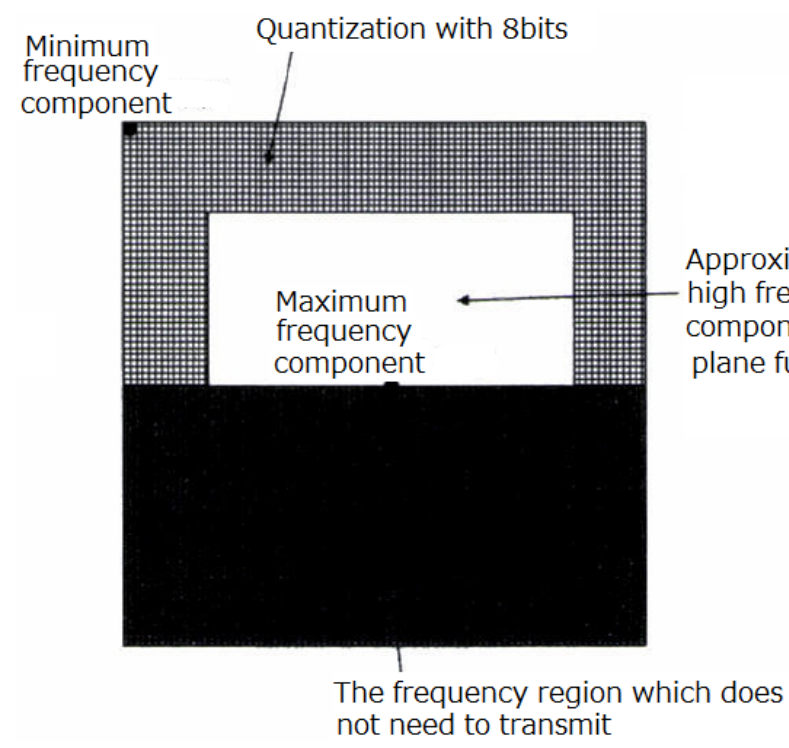

Fig. 3. Coding Method for the Parameters of the S/N Ratio. 


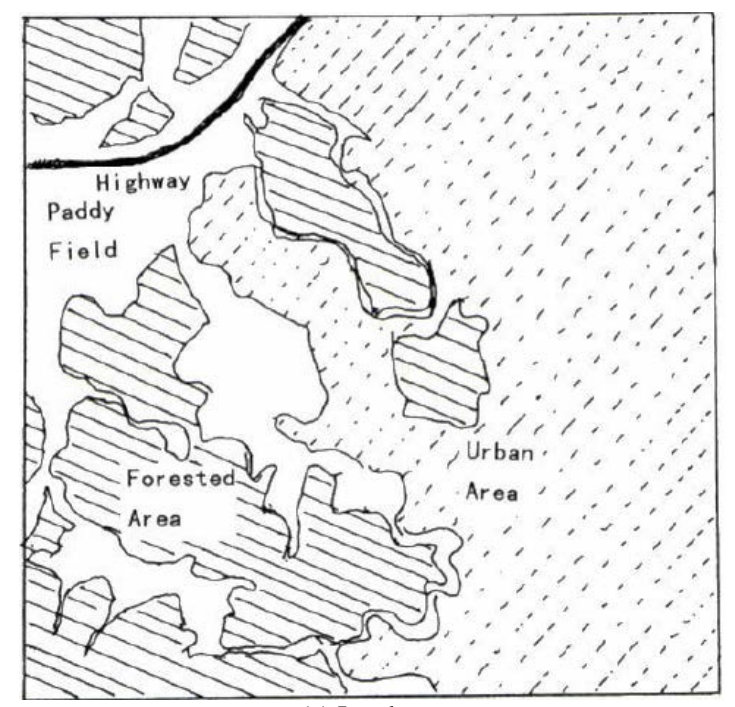

(a) Land use.

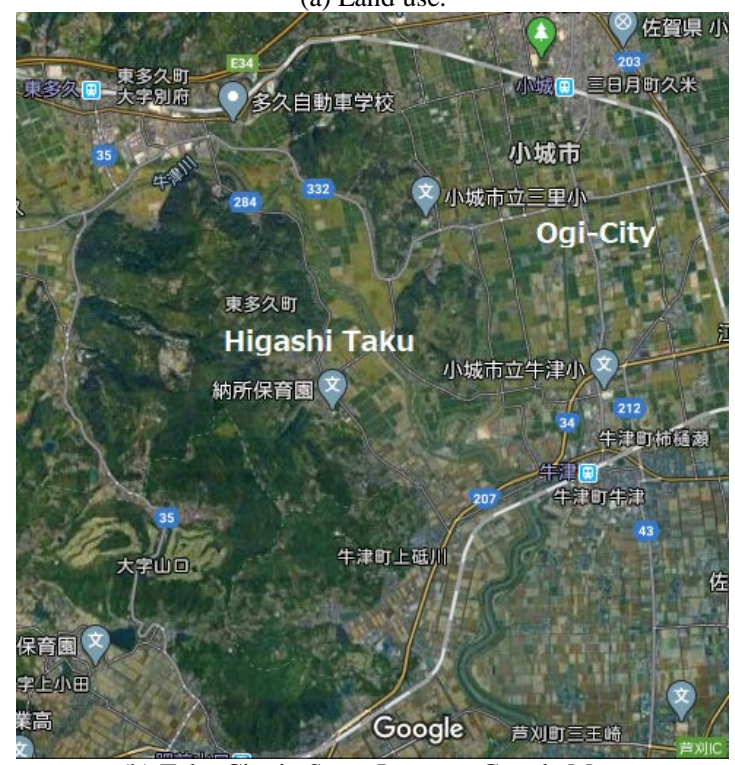

(b) Taku City in Saga, Japan on Google Map.

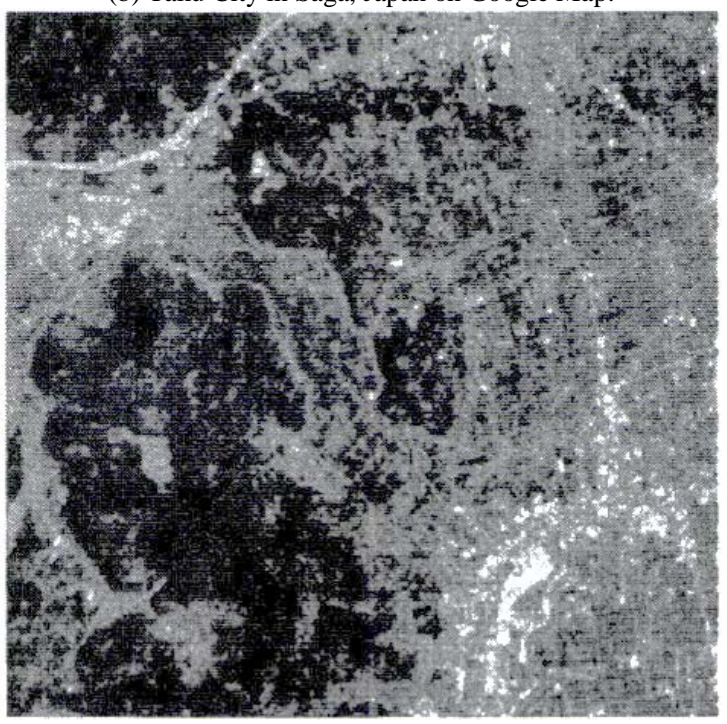

(c) Landsat TM Image of Saga Japan.

Fig. 4. Original Image of Taku City in Saga Observed from Landsat.

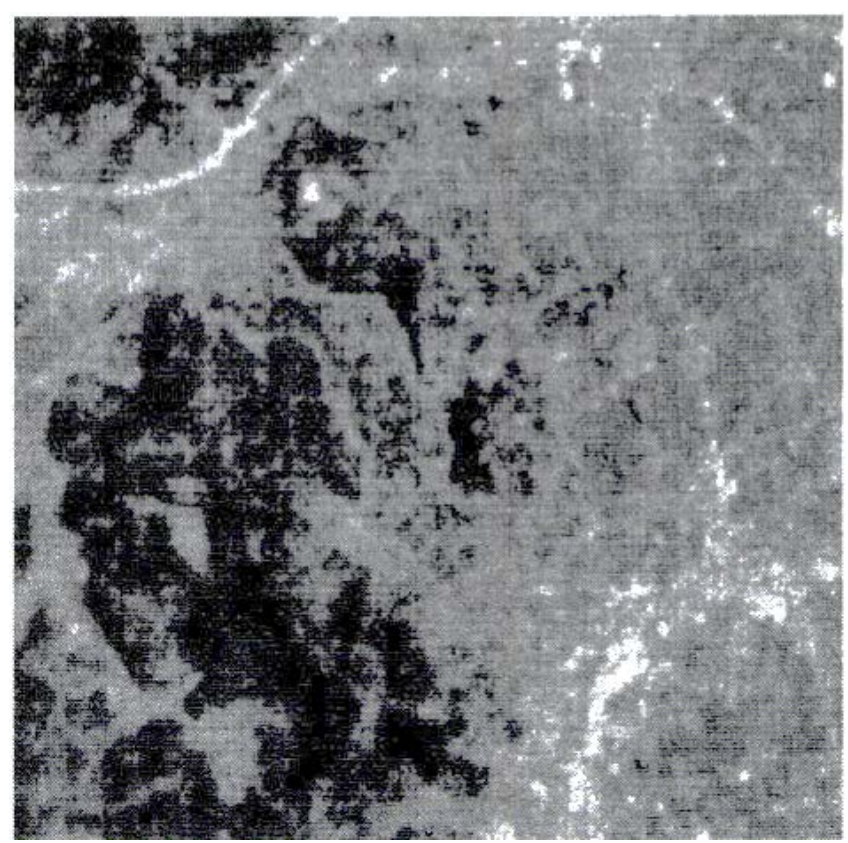

Fig. 5. Compressed Image with DCT Compression (Compression Factor=69).

When finding the regression plane, if data $\left(x_{1}, y_{1}\right),\left(x_{2}\right.$, $\left.y_{2}\right), \ldots,\left(x_{n}, y_{\mathrm{n}}\right)$ are obtained:

$\mathrm{y}=\mathrm{a}_{0}+\mathrm{a}_{1} \mathrm{x}_{1}+\ldots+\mathrm{a}_{\mathrm{n}-1} \mathrm{x}_{\mathrm{n}-1}+\mathrm{a}_{\mathrm{n}} \mathrm{x}_{\mathrm{n}}$

The sum of squares of the error

$\mathrm{J}=\Sigma\left\{\mathrm{y}_{\mathrm{i}-}\left(\mathrm{a}_{0}+\mathrm{a}_{1} \mathrm{x}_{1 \mathrm{i}}+\ldots+\mathrm{a}_{\mathrm{n}-1} \mathrm{x}_{(\mathrm{n}-1) \mathrm{i}}+\mathrm{a}_{\mathrm{n}} \mathrm{x}_{\mathrm{ni}}\right)\right\}^{2}$

These are determined so that is minimized. If both sides are differentiated by $a_{0}, \ldots, a_{n}$ and set to 0 , the following normal equation is obtained:

$\frac{\partial J}{\partial a_{0}}=-2 \sum\left\{y_{i}-\left(a_{0}+a_{1} x_{1 i}+\cdots+a_{n} x_{n i}\right)\right\}$

$\frac{\partial J}{\partial a_{1}}=-2 \sum x_{1 i}\left\{y_{i}-\left(a_{0}+a_{1} x_{1 i}+\cdots+a_{n} x_{n i}\right)\right\}$

$\frac{\partial J}{\partial a_{n}}=-2 \sum x_{1 n}\left\{y_{i}-\left(a_{0}+a_{1} x_{1 i}+\cdots+a_{n} x_{n i}\right)\right\}$

By solving this, the coefficients $a_{0}, \ldots, a_{n}$ are obtained, and only the coefficients are transmitted. When actually performing approximation, a three-dimensional plane is considered, so calculation is performed with $n=2$. Furthermore, since the $\mathrm{S} / \mathrm{N}$ ratio of the low frequency component is a floating point number, quantization is performed with 8 bits per element in order to reduce the capacity as much as possible. That is, one element is represented by one byte.

The quantization method first finds the maximum value from the low frequency components, sets it to MAX, and sets the minimum value at the boundary between the low frequency component and the high frequency component to MIN. Next, quantization is performed so that MAX becomes 255 and MIN becomes 0.

Furthermore, since this $\mathrm{S} / \mathrm{N}$ ratio is a value in the frequency domain, there is a property that the same value 
appears at a position symmetric with respect to the highest frequency component. In particular, with respect to the imaginary part, a value whose polarity is reversed appears at a position symmetrical with respect to the highest frequency component. By utilizing this property, the capacity can be further reduced by half.

These series of parameterization processes are performed on the transmission side, the low frequency component of the $\mathrm{S} / \mathrm{N}$ ratio is inversely quantized on the reception side, and for high frequency components, a plane equation is obtained from the transmitted coefficients. It is possible to calculate the actual value.

Fig. 6 shows the result of approximation of Fig. 3.

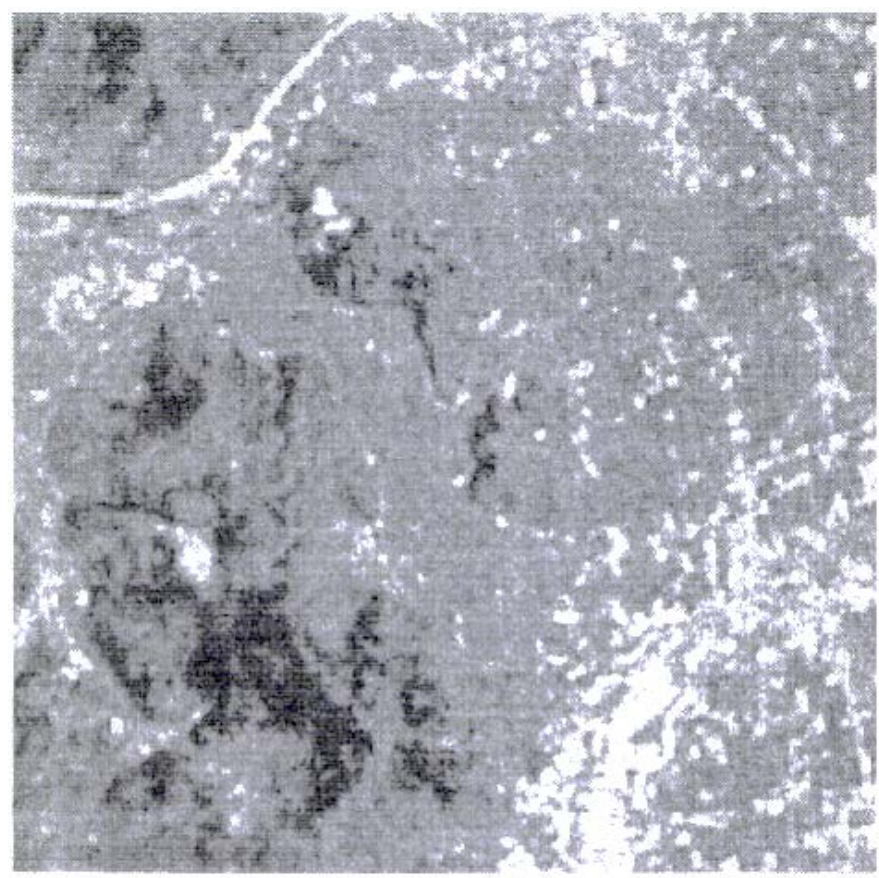

Fig. 6. Restored Image with Approximation of S/N Ratio.

\section{EXPERIMENTS}

\section{A. Original Image}

Fig. 4 shows the original image. The sample image used this time is a Landsat / TM image near Ogi, Taku in the western part of Saga city, and is a PPM image using images of blue, green, and red wavelengths. The image size is $256 \times 256$ pixels. There are various formats of image data, but there are PPM format (color image) and PGM format (black and white image) as uncompressed formats. The luminance value of one pixel is represented by 8 bits for PGM and 24 bits for PPM (8 bits for red, 8 bits for green, and 8 bits for blue). Image data is recorded with 1 byte per pixel for PGM and 3 bytes per pixel for PPM, each with a header of about 15 to 30 bytes.

In PGM, since one pixel is one byte, the number of pixels of an image becomes the capacity of image data almost as it is. If the image size is $512 \times 512$, the data capacity will be about 262144 bytes, which is a considerable capacity. If this is a color image, the number of bits per pixel will change from 8 bits to 24 bits, so it will triple to 786,432 bytes, further expanding the capacity. If the moving image is a color moving image, a large amount of still images will be included, so that the capacity will be further increased.

Fig. 5 shows an image compressed by the discrete cosine transform. This is a compressed version. The $\mathrm{Q}$ factor is specified by an integer value in the range of 0 to 100 , with 100 being the best image quality and 0 being the worst. The $\mathrm{Q}$ factor $=10$ specified here is a considerably high compression ratio.

In this case, the capacity of the original image was 196720 bytes, the capacity of the compressed data was 2869 bytes, and the compression ratio was about 69 times. Figure 6 shows the result of creating and restoring a Wiener filter without creating an SN ratio at all. It can be seen that the details have been restored.

\section{B. Compression with Image Restoration}

Fig. 6 shows the results of an attempt to restore the image by constructing a Wiener filter from the approximated $\mathrm{S} / \mathrm{N}$ ratio. Although it was a slightly blurred image, it was able to be restored in considerable detail.

The image quality after restoration was improved compared to that before restoration, and it was found that restoration of high frequency components was possible to some extent. The data capacity of the S / N ratio is 3816050 bytes without approximation, and has a capacity of about 3.8 Mbytes. In this data format, the SN ratio for each frequency component is represented by a floating point number and output as text data.

As a result of the approximation, the data capacity of the $\mathrm{SN}$ ratio was 5053 bytes, and the compression was remarkable. After all, when the compressed image and the decompression parameters (encoded data of S / $\mathrm{N}$ ratio) were combined, the capacity became 7922 bytes and the compression ratio became 24.83 times. At this time, it was also found that when trying to obtain the same image quality by compression with discrete cosine transform, the compression ratio could only be obtained about 20 times. This is slightly less than JPEG compressed with a $\mathrm{Q}$ factor of 98. Comparing the image quality, it is not worse than the one compressed with $\mathrm{Q}$ factor $=98$.

\section{SOME Discussions}

The image was compressed at a fairly high compression rate by the discrete cosine transform, and a filter was created to correct the degraded image and restore a good quality image. Then, the data ( $\mathrm{S} / \mathrm{N}$ ratio) necessary to construct the filter was parameterized and considered to be transmitted together with the compressed data.

As a result, it was possible to obtain an image that was somewhat blurry but was quite close to the original image. The original image used this time has a capacity of about 196 Kbytes, the data capacity of the compressed image $(\mathrm{Q}$ factor $=$ 10 ) is 2869 bytes, and the capacity of the restoration filter is about 3.6 Mbytes (red 1.2 bytes, green 1.2 Mbytes, blue 1.2 Mbytes) Bytes), but with the approximation of the SN ratio, the capacity of the restoration filter could be compressed to about 53 Kbytes. 
If this is added to the capacity of the compressed image of 2869 bytes, the compression rate will be about 7 times at about $56 \mathrm{~K}$ bytes, which is slightly less than that of JPEG compressed with $\mathrm{Q}$ factor $=98$, and the image quality is not worse than that. The Wiener filter created this time was able to completely restore the original image, but even if the $\mathrm{S} / \mathrm{N}$ ratio for constructing the Wiener filter was approximated, an image close to the original image could be obtained.

The compression ratio was relatively effective, about 25 times, and the effect on the image quality by approximation of the $\mathrm{S} / \mathrm{N}$ ratio was also small. It was found that if the same image quality was to be obtained by a compression method involving discrete cosine transform, the compression ratio would be about 20 times, and the compression effect would be reduced by $25 \%$. This is the result of a subjective evaluation experiment of image quality by a one-to-one comparison method based on the Thurston method.

Prepare a compressed image in which the Q factor in JPEG compression is changed in 10 steps from 10 to 90 (the compression ratio changes from about 90 times to 5 times), and compare the compressed image proposed this time with the one-to-one comparison This is the result of evaluating the quality of image quality for 40 subjects. Fig. 7 shows an image with a compression ratio of 20 at this time and an image quality determined to be comparable to that of the proposed method.

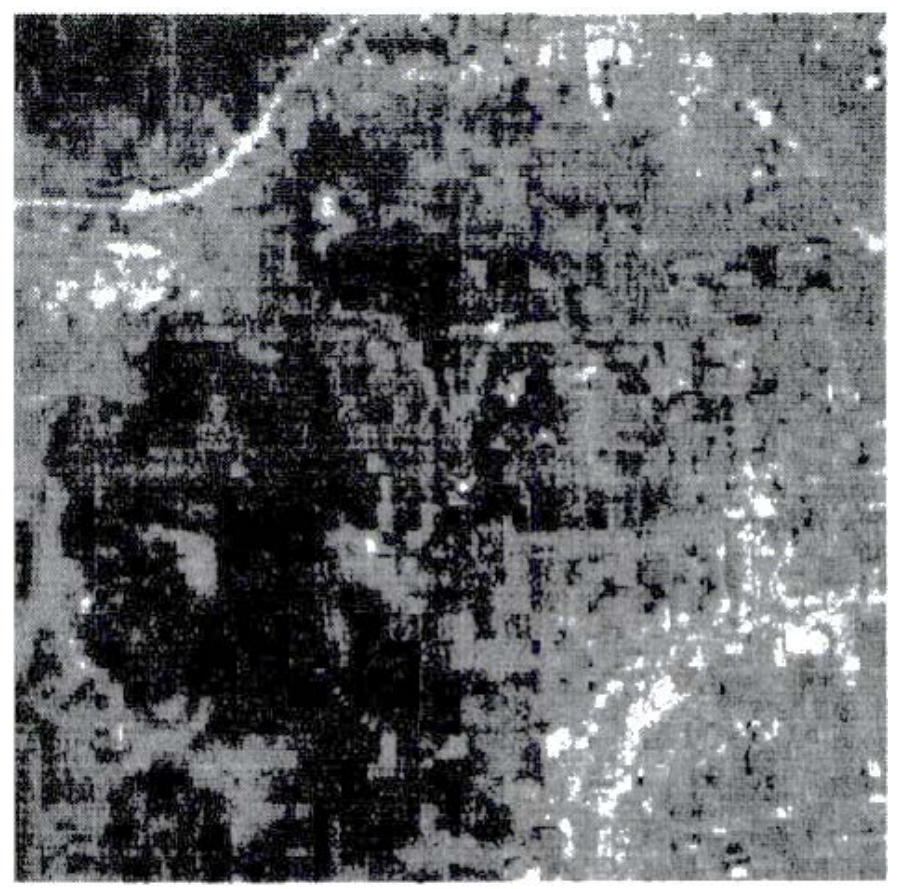

Fig. 7. Compressed Image with JPEG Compression (Compression Factor=69).

\section{CONCLUSION}

Discrete Cosine Transformation: DCT based image data compression considering image restoration is proposed. An image data compression method based on the compression (DCT) featuring an image restoration method is proposed. DCT image compression is widely used and has four major image defects. In order to reduce the noise and distortions, the proposed method expresses a set of parameters for the assumed distortion model based on an image restoration method.

The results from the experiment with Landsat TM (Thematic Mapper) data of Saga show a good image compression performance of compression factor and image quality, namely, the proposed method achieved $25 \%$ of improvement of the compression factor compared to the existing method of DCT with almost comparable image quality between both methods.

\section{FUTURE RESEARCH WORKS}

Further research works are required for the applicability of the proposed data compression method with the other remote sensing images.

\section{ACKNOWLEDGMENT}

The author would like to thank Prof. Dr. Hiroshi Okumura and Prof. Dr. Osamu Fukuda of Saga University for their valuable comments and suggestions.

\section{REFERENCES}

[1] Ono and Suzuki, "Easy-to-understand JPEG / MPEG2 Implementation", p.1-p.93 Ohmsha 1995.

[2] Supervised by Harashima, " Image Information Compression ", Ohmsha 1994.

[3] Gregory K. Wallace, gThe JPEG Still Picture Compression Standardh, Submitted in December 1991 for publication in IEEE Transactions on Consumer Electronics.

[4] Co-authored by K.R.Rao / P.Yip, translated by Hiroshi Yasuda and Hiroshi Fujiwara, "Image Coding Technology-DCT and Its International Standards", Ohmsha 1993.

[5] Takagi, Shimoda, and Arai, "Image Analysis Handbook", University of Tokyo Press 1991.

[6] K. Arai, T. Yamasaki and Y. Terayama, A method for image compression with a cosmetic restoration (COSRES Coding), Proceedings of the Australasian Conference on Remote Sensing, Mar., 1994.

[7] K. Arai, Yamazaki, Terayama, "Data Compression with Image Restoration", 1994 Annual Conference of the Institute of Television Engineers of Japan, pp.197-198, July 1994.

[8] N. P. Galatsanos, R. T. Chin, gDigital Restoration of Multichannel Imageh IEEE Trans. on Acoust., Speech and Signal Processing, ASSP37, pp.415-421, Mar., 1989.

[9] Fujimoto, Fujita, Yoshida, "Reconstruction from Multiple Degraded Images Based on Image Probability Model" IEICE Technical Report, EID97-137, IE97-162, pp.15-22, Feb., 1998.

[10] Masao Ikuzawa, " Introduction to Statistics for Psychology, " Minerva Shobo, 1975.

[11] Y,Yasuda and Kohei Arai, Facsimile data compression by rearranging picture elements, Proc.of the 7th Picture Coding Symposium, S405, 4546,1977.

[12] Kohei Arai, Data compression for archiving of ADEOS data, Proc.of the IGARSS'89, D3-5, 914-918, 1989.

[13] Kohei Arai, T.Yamasaki and Y.Terayama Method for image compression with a cosmetic restoration, Proc.of the Australasian Conference on Remote Sensing, 1993.

[14] Arai,K., T.Yamasaki and Y.Terayama, A method for image compression with cosmetic restoration, Proceedings of the 9th Australasian Conference on Remote Sensing, Mar.1994.

[15] S. Sobue, K.Cho and Kohei Arai, A Study of Data Lossy Compression Using JPEG/DCT and Fractal Method, Proceedings of the ISTS Symposium in Gifu, May 1996. 
[16] Kohei Arai, Preliminary Study on Information Lossy and Lossless Coding of Data Compression for Archiving ADEOS Data, IEEE Trans. on Geoscience and Remote Sensing, Vol.28, No.4, pp.732-735, Jul.1990.

[17] Kohei Arai, Method for video data compression based on space and time domain seam carving maintaining original quality when it is replayed, International Journal of Research and Reviews on Computer Science, 2, 4, 1063-1068, 2011.

[18] Kohei Arai, Yuji Yamada, Data hiding method which robust to runlength data compression based on lifting dyadic wavelet transformation, Proceedings of the 11th Asian Symposium on Visualization, ASV-1108-11, 1-8, 2011.

[19] Kohei Arai, Method for image portion retrieval and display for comparatively large scale of imagery data onto relatively small size of screen which is suitable to block coding of image data compression, International Journal of Advanced Computer Science and Applications, 4, 2, 218-222, 2013.

[20] Kohei Arai, Prediction method of El Nino Southern Oscillation event by means of wavelet based data compression with appropriate support length of base function, International Journal of Advanced Research in Artificial Intelligence, 2, 8, 16-20, 2013.

[21] Kohei Arai, Method for data hiding based on Legall 5/2 (CohenDaubechies-Feauveau: CDF 5/3) wavelet with data compression and random scanning of secret imagery data, International Journal of
Wavelets Multi Solution and Information Processing, 11, 4, 1-18, B60006 World Scientific Publishing Company, DOI: I01142/SO219691313600060, 1360006-1, 2013.

\section{AUTHOR's PROFILE}

Kohei Arai, He received BS, MS and PhD degrees in 1972, 1974 and 1982, respectively. He was with The Institute for Industrial Science and Technology of the University of Tokyo from April 1974 to December 1978 also was with National Space Development Agency of Japan from January, 1979 to March, 1990. During from 1985 to 1987, he was with Canada Centre for Remote Sensing as a Post Doctoral Fellow of National Science and Engineering Research Council of Canada. He moved to Saga University as a Professor in Department of Information Science on April 1990. He was a councilor for the Aeronautics and Space related to the Technology Committee of the Ministry of Science and Technology during from 1998 to 2000 . He was a councilor of Saga University for 2002 and 2003. He also was an executive councilor for the Remote Sensing Society of Japan for 2003 to 2005. He is a Science Council of Japan Special Member since 2012. He is an Adjunct Professor of University of Arizona, USA since 1998. He also is Vice Chairman of the Science Commission "A" of ICSU/COSPAR since 2008 then he is now award committee member of ICSU/COSPAR. He wrote 55 books and published 620 journal papers as well as 450 conference papers. He received 66 of awards including ICSU/COSPAR Vikram Sarabhai Medal in 2016, and Science award of Ministry of Mister of Education of Japan in 2015. He is now Editor-in-Chief of IJACSA and IJISA. http://teagis.ip.is.sagau.ac.jp/index.html 